\title{
Recent advances in organoid culture for insulin production and diabetes therapy: methods and challenges
}

\author{
Ahmed Abdal Dayem, Soo Bin Lee, Kyeongseok Kim, Kyung Min Lim, Tak-il Jeon \& Ssang-Goo Cho* \\ Department of Stem Cell \& Regenerative Biotechnology, Konkuk University, Seoul 05029, Korea
}

Breakthroughs in stem cell technology have contributed to disease modeling and drug screening via organoid technology. Organoid are defined as three-dimensional cellular aggregations derived from adult tissues or stem cells. They recapitulate the intricate pattern and functionality of the original tissue. Insulin is secreted mainly by the pancreatic $\beta$ cells. Large-scale production of insulin-secreting $\beta$ cells is crucial for diabetes therapy. Here, we provide a brief overview of organoids and focus on recent advances in protocols for the generation of pancreatic islet organoids from pancreatic tissue or pluripotent stem cells for insulin secretion. The feasibility and limitations of organoid cultures derived from stem cells for insulin production will be described. As the pancreas and gut share the same embryological origin and produce insulin, we will also discuss the possible application of gut organoids for diabetes therapy. Better understanding of the challenges associated with the current protocols for organoid culture facilitates development of scalable organoid cultures for applications in biomedicine. [BMB Reports 2019; 52(5): 295303]

\section{INTRODUCTION}

Diabetes mellitus (DM) is a well-known disease increasing the risk of morbidity and mortality due to disease complications. Current therapies do not result in complete recovery. The lack of insulin production due to $\mathrm{T}$ cell-associated autoimmune damage of pancreas $\beta$ cells is the main cause of type $1 \mathrm{DM}$. Therefore, efficient therapeutic strategies designed to restore insulin production are needed.

Transplantation of human islets into the portal vein to replace the damaged $\beta$ cells resulted in regulation of blood glucose levels and significant improvement in the patient

${ }^{*}$ Corresponding author. Tel: +82-2-450-4207; Fax: +82-2-450-4207; E-mail: ssangoo@konkuk.ac.kr

https://doi.org/10.5483/BMBRep.2019.52.5.089

Received 12 March 2019

Keywords: Insulin secretion, Organoids, Pluripotent stem cells, Scaffolds, Three-dimensional (3D) health condition $(1,2)$. However, this method is limited by the shortage of islet donors and the need to induce immunosuppression to prevent immune rejection (3). Moreover, intricate mechanisms such as redifferentiation and dedifferentiation are prerequisites for the expansion of mature $\beta$ cells $(4,5)$. Accordingly, application of pluripotent stem cells (PSCs) including embryonic stem cells (ESCs) and induced pluripotent stem cells (iPSCs) is one of the leading strategies for the generation of $\beta$ cells with alternative function due to the high capacity of PSCs to differentiate into a wide range of lineages (6). Various protocols generate $\beta$ cells from PSCs following treatment with signal-modulating compounds, hormones, and growth factors that induce multi-step pancreatic endoderm (PE) differentiation (7-9). The elevated expression of $\beta$ cell-specific transcription factors and insulin secretion following glucose challenge guarantee authentic $\beta$ cells (10). Ectopically transplanted PSCs-derived pancreatic $\beta$ cells in immunodeficient diabetic mice triggered insulin secretion upon glucose challenge (11). However, generation of pancreatic $\beta$ cells from PSCs is labor-intensive, timeconsuming, expensive, and inefficient, and the clinical applications of PSCs are restricted by biosafety issues (12). Notably, the conversion of duct and islet cells into cells similar to mesenchymal cells is the main obstacle hindering the expansion of pancreatic cells in the monolayer platform $(4,13$, 14). Therefore, the development of a new system based on three-dimensional (3D) organoid culture is essential for efficient differentiation and maturation of pancreatic $\beta$ cells, as it simulates the inherent cellular environment (cell polarization, alignment, and niches) (15). Here, we will discuss methods of pancreatic organoid culture and their challenges along with its impact on the large-scale generation of PSCs-derived pancreatic $\beta$ cells. Further, we discuss the possible application of gut organoids for regeneration of damaged pancreatic cells in diabetes therapy.

\section{OVERVIEW OF ORGANOIDS}

Organoid culture or organs-in-a-dish is the in vitro simulation of normal organs and their structure and function. It requires 3D growth of PSCs or other primary cells. During the organoid culture, the stem cells aggregate and differentiate in response to biophysical cues resulting in complex cellular structures that 
imitate the structure and function of the mature tissue (16-18). Organoid culture is a breakthrough technology intended to investigate organ development, pathogenicity, disease models, and drug discovery.

In 2009, the Clevers research group demonstrated the organoid culture for the first time, using stem cells derived from the intestine, which laid the initial groundwork in the organoid era (19) followed by the development of optic cup from ESCs (20). Subsequently, a series of studies investigating organoid culture using various cells derived from liver, kidney, pancreas, brain, stomach, and prostate have emerged (21-26). Here, we focus on pancreatic and gut organoids and their application in diabetes therapy.

\section{PANCREATIC DEVELOPMENT}

Pancreas is an organ manifesting both endocrine and exocrine functions and plays a crucial role in diseases such as diabetes, pancreatic cancer, and disease associated with pancreatic inflammation. The exocrine function is attributed to acinar function, which is associated with the secretion of digestive enzymes, whereas the endocrine function is related to epithelial clusters (islets of Langerhans) including $\alpha, \beta, \delta, \varepsilon$ and pancreatic polypeptide (PP; formerly known as $\gamma$ ) cells, which secrete glucagon, insulin, somatostatin, ghrelin, and pancreatic polypeptide, respectively (27).

Pancreatic development occurs concomitantly in the ventral as well as the dorsal anterior foregut endoderm (on embryonic day 9.5 (E9.5)), followed by expansion into the surrounding mesenchyme and proliferation, differentiation, and branching resulting in mature organ formation (28-31). The early-stage multipotent pancreatic progenitor cells, which express Sry (sex-determining region Y)-like box 9 (Sox9), hepatocyte nuclear factor 1 homeobox $\mathrm{B}(\mathrm{Hnf1B})$, pancreas transcription factor 1 subunit alpha (Ptf1A), and pancreatic and duodenal homeobox $1(\mathrm{Pdx} 1)$, are developed before $\mathrm{E} 11.5$, and generate all the pancreatic cells including endocrine lineages, exocrine cells (acinai), and ductal cells subsequently $(32,33)$. The two progenitor cells generated by E14.5 yield a limited range of pancreatic cells; at this stage, Sox9-positive cells generate acini (34).

Fibroblast growth factor (FGF) and Notch (named after the appearance of a notch in the wings of mutant Drosophila melanogaster) signaling pathways play key roles in pancreatic development. For instance, the decrease in the activity of FGF10 is correlated with the differentiation of endocrine cells (35). In addition, the activation of Notch1 signaling resulted in undifferentiated status and suppressed differentiation of pancreatic precursor cells $(36,37)$.

\section{PANCREATIC ORGANOID CULTURE}

Organoids derived from pancreatic tissues

In 2013, Greggio et al. set up artificial niches for the in vitro
3D development of pancreas into organoids with sustained expansion up to 2 weeks and successful differentiation into duct, acini, and endocrine cells (38). In this study, the organoids were cultured using epithelial cells isolated from the pancreas of E10.5 mouse embedded in growth factor-reduced Matrigel, the trade name for a gelatinous protein mixture secreted by Engelbreth-Holm-Swarm (EHS) mouse sarcoma cells, and with organogenesis medium, composed of DMEM/Nutrient Mixture F12-supplemented with 10\% KnockOut Serum Replacement, phorbol myristate acetate, 2-mercaptoethanol, Y-27632 (Rho-associated protein kinase (ROCK) inhibitor), epidermal growth factor (EGF), mouse R-spondin (a secreted protein encoded by the Rspo1 gene interacting with WNT/B-catenin signaling), FGF1, FGF10, and heparin. The authors also fabricated chemically defined matrices such as synthetic hydrogels and compared their effects with the Matrigel (38). The authors found that polyethylene glycol (PEG)-based hydrogels led to efficient organoid expansion and covalent functionalization with laminin. Furthermore, soft hydrogels showed superior activity in maintaining cluster formation and progenitors compared with the stiff hydrogel (38).

In 2018, Takahashi et al. developed an efficient protocol for the in vitro preparation of islet-like organoids with vascularization using a self-condensation culture system (39). Self-condensation culture is based on co-culture of various types of lineages with vasculatures, and this technique facilitates the in vitro investigation of events during early organogenesis (40). Accordingly, the authors co-cultured the MIN6 (dissociated $\beta$ cell lines) with human vascular endothelial cells (HUVECs) and mesenchymal stem cells (MSCs) on a 3D platform by embedding in Matrigel (39). This vasculature is essential for providing oxygen and nutrients as well as enhancing angiogenesis-related signaling for further growth and differentiation. The co-culture with the stromal cells boosts insulin production by MIN6 cells, as reported previously by Takebe et al. (41). Transplantation of the generated organoid in diabetic mice efficiently alleviated hypoglycemia (39).

The reprogramming of acinar cells into pancreatic endocrine $\beta$ cells with the transcription factors regulating endocrine formation, namely neurogenin-3 (Neurog3 or Ngn3; a basic helix-loop-helix (bHLH) transcription factor), Pdx1, and V-maf musculoaponeurotic fibrosarcoma oncogene homolog A (MafA) represents an important alternative source to regenerate the damaged $\beta$ cells (42). In this regard, the overexpression of Ngn3, Pdx1, and MafA in mouse ductal pancreatic organoids using lentiviral vectors resulted in the generation of pancreatic $\beta$-like cells that showed a robust resemblance to $\beta$ cells in insulin secretion and transcriptome-wide analysis (43). Interestingly, the phospho-mutant form of Ngn3 together with $\operatorname{Pdx} 1$ and MafA markedly promoted the expression of $\beta$ cell-associated genes and showed a four-fold increase in reprogramming toward insulin-positive endocrine cells 
compared with the wild-type Ngn3 (43). The phospho-mutant form of Ngn3 was prepared via replacement of serine with alanine and therefore, cannot be phosphorylated with cyclin-dependent kinases (CDKs). Therefore, the Ngn3 mutation via mutations associated with the phosphorylation site resulted in enhanced endocrine cell reprogramming for ductal organoids.

In 2018, Loomans et al. devised an efficient 3D culture system for the expansion of human adult pancreatic tissue, yielding subpopulations exhibiting the characteristics of progenitor cells with potential for endocrine differentiation (44). Islet-voided pancreatic tissues were enzymatically isolated from normal and diabetic donors and were embedded in Matrigel and maintained in an expansion medium supplemented with EGF/Noggin (also known as NOG and a slang word for "head")/R-spondin. After 3 days of culture, budding structures were detected in more than $90 \%$ of the pancreatic aggregates and cauliflower-like morphology was observed by day 7 (passage 0). On day 7 , the formed organoids exhibited a ductal phenotype confirmed by the positive expression of an epithelial marker, cytokeratin 19 (KRT19, CK-19, or K19 encoding a 40 kDa type 1 keratin) detected in cell linings. This phenotype was still observed until passage 3 without any insulin-, glucagon-, or amylase-positive cells. Fetal or adult pancreatic tissue-derived organoids showed a high expression of aldehyde dehydrogenase (ALDH) in the tips of the budding regions. Notably, cells expressing high levels of ALDH carry pancreatic progenitor markers, such as PDX1, carboxypeptidase A1 (CPA1), PTF1A, and MYC, compared with cells displaying low ALDH expression. These results are in accordance with the findings of Zhou et al., in which increased expression of progenitor markers was detected in ALDH-highly expressed cell populations (45). Furthermore, the derived organoids (passage 0) exhibited endocrine differentiation, and showed downregulation of $\mathrm{ALDH}$ and proliferation markers concomitant with the increase in insulin-positive cell populations. One month after the transplantation of the organoids into immunodeficient mice, insulin-positive cells and circulating C-peptide were detected. In 2019, Jiang et al. developed novel 3D physiomimetic extracellular matrix (ECM) hydrogels for the encapsulation of human as well as rat islets (46). The 3D physiomimetic ECM hydrogels were prepared via decellularization of the porcine tissues (pancreas, bladder, and lung), via alternative incubation in hypertonic and hypotonic solutions. These hydrogels supported the long-term culture of human and rat islets and also maintained insulin secretion in response to glucose treatment (46). Interestingly, the authors demonstrated the formation of fibroblast-like protrusions from the spheroids, for the migration of endothelial cells. The effect of 3D physiomimetic ECM hydrogel on the generation of mature pancreatic $\beta$ cells needs further investigations.

Further details of the organoid cultures derived from adult and fetal pancreatic tissues are discussed and summarized elsewhere (47).

\section{Organoids derived from PSCs}

Previous studies demonstrated the role of organoid cultures derived from PSCs on the maturation and functionality of various cells $(48,49)$.

In 2015, Shim et al. generated 3D islet-like aggregates from hESCs, which efficiently regulated the glucose level in diabetic mice (50). In this study, the authors applied a step-wise protocol based on the application of various small molecule inhibitors and growth factors. Interestingly, the authors showed for the first time that the inhibition of MSCs populations within the $\mathrm{hESC}$-derived $\mathrm{PDX}^{+}$aggregates following the treatment of proteoglycan sulfation inhibitors as well as Notch signaling inhibitors resulted in prompt maturation of hESC-derived pancreatic progenitors cells, suggesting upregulation of Ngn3 and neurogenic differentiation 1 (NeuroD1; also called $\beta 2$, a bHLH protein) (50). The transplantation of hESC-derived aggregates into streptozotocin (STZ; a naturally occurring alkylating anti-neoplastic agent that is particularly toxic to the pancreatic $\beta$ cells)-induced diabetic mice resulted in significant improvement in hyperglycemia (50).

In 2016, Kim et al. generated islet-like organoids from hESCs, which efficiently produced insulin upon glucose challenge (51). In this study, the authors devised a step-wise protocol for the generation of hormone-expressing pancreatic endocrine cells. Towards this end, the hESCs were initially differentiated into a definitive endoderm (DE) via treatment with GSK3ß-inhibitors, such as CHIR99021 and $\mathrm{LiCl}$, and activin $A$ (a type of disulfide-linked dimeric activin proteins

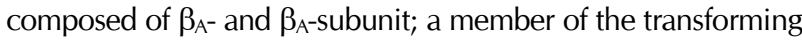
growth factor beta (TGF- $\beta$ ) family of proteins) (Fig. 1A). PE induction occurred by further exposing the cells to retinoic acid (RA; a metabolite of vitamin A (retinol)), KAADcyclopamine (a cell-permeable analog of cyclopamine that specifically inhibits the hedgehog (Hh) signaling), SB432942, dorsomorphin (a potent and selective inhibitor of the bone morphogenetic protein (BMP) signaling pathway and a selective and reversible inhibitor of the AMP-activated protein kinase (AMPK)), and basic FGF (bFGF) for 6 days. PE differentiated into endocrine progenitor (EP) cells in 4 days before ultimate differentiation into endocrine cells (EC) in additional 4 days. DE differentiation was confirmed by the elevated expression of CXCR4 in flow cytometry (FACS) analysis and increased expression of mRNA and protein expression of DE-associated markers, namely FOXA2, SOX17, and GATA4. The PE stage was detected depending on the high expression of PDX1, SOX9, and HNF1 $\beta$. The EP stage, an intermediate stage between $\mathrm{PE}$ and $\mathrm{EC}$ stages, was verified by the high expression of Ngn3 co-expressed with PDX1 and NKK2.2 (detected in the nucleus). At the level of transcription, EP cells showed a high expression of Ngn3 and NKK2.2 and the expression of PDX1 was lower than that of the PE cells. 
A

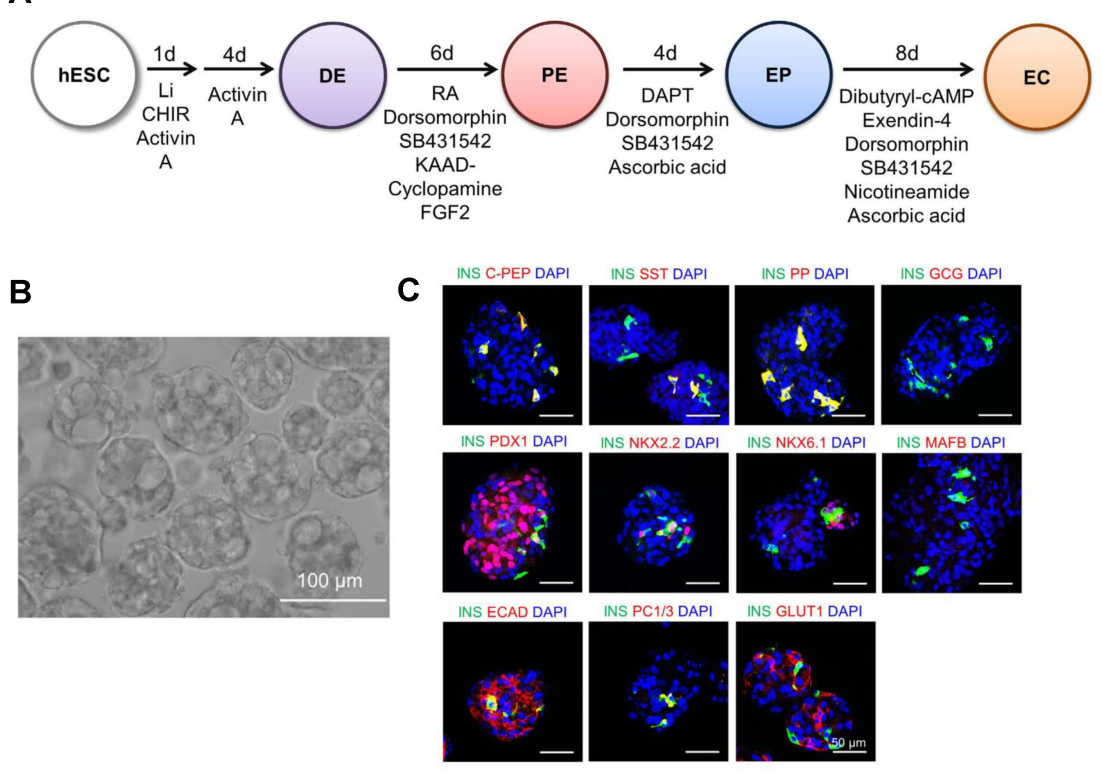

Fig. 1. Step-wise protocol elucidating the endocrine differentiation of hESCs and the characterization of hESC-ECCs. (A) Experimental protocol outlining the stages, and inducing factors and chemicals for differentiation of hESCs into definitive endoderm (DE), pancreatic endoderm $(\mathrm{PE})$, and endocrine progenitor (EP) cells and the final insulin-producing endocrine cells (EC). (B) Phase-contrast image of hESC-ECCs. Scale bar $=100 \mu \mathrm{m}$. (C) Immmunocytochemical analysis showing the expression of $\beta$ cell-associated genes, endocrine hormone genes, and $\beta$ cell function-linked proteins. Scale bar $=50$ $\mu \mathrm{m}$. The figure is reproduced from the article by Kim et al. 2016 (51). This work is licensed under a Creative Commons Attribution 4.0 International License (http://creativecommons.org/licenses/by/ 4.0/).
The EC cells showed an increased expression of pancreatic endocrine hormones including insulin (INS), pancreatic polypeptide (PP), and somatostatin (SST). Moreover, the de novo insulin synthesis was shown by the co-expression of C-peptide with insulin. ECs expressed the $\beta$ cell-related transcription factors, such as PDX1, NKX2.2, NKX6.1, and MAFB. However, the authors detected a low expression of the mature $\beta$ cell-associated marker (NKX6.1), which indicates the immaturity of the derived ECs. The authors clustered the derived ECs into pancreatic islet-like structures and the ECs were dissociated and plated onto an uncoated 96-well plate under static conditions. Subsequently, the EC clusters (ECC) developed after one day (Fig. 1B) and express mature $\beta$ cell-associated transcription factors (PDX1, NKX6.1, and MAFA), glucose sensor genes (SLC2A1 and GCK), gap-junction genes (CDH1 and $\mathrm{CX} 36)$ and endocrine hormones (Fig. 1C). In addition, the co-expression of insulin with NKK6.1 was observed. Notably, ECCs showed a high expression of E-cadherin (ECAD) and glucose transporter 1 (GLUT1) in the membrane (51). Therefore, the clustering of ECs resulted in significant maturation of pancreatic $\beta$ cells suggesting the formation of islet-like organoids. The hESC-derived ECCs showed high insulin secretion mediated by $\mathrm{K}_{\text {ATP }}$ channels. Upon glucose stimulation, ECC secreted increased levels of C-peptide (51) and the in vitro insulin secretion after glucose exposure again confirmed the maturity and the functionality of $\beta$ cells. In addition, the transplantation of hESC-derived ECCs into STZ-treated mice significantly modulated the blood glucose level shortly after the transplantation (3 days).
However, the transplanted hESC-derived ECCs showed transient improvement in blood glucose level after the transplantation (only 12 days) (51). Accordingly, further improvements in methodology for obtaining ECCs from PSCs are required to prolong the in vivo functionality.

In 2009, Wang et al. reported a 3D differentiation method for the generation of islet-like insulin-secreting cells derived from mouse ESCs (mESCs) (52). The mESCs were mixed with neutralized type I rat tail collagen solution (supplemented with a mixture of basement membrane proteins plus 2 DMEM) and then layered onto a collagen-coated plate and incubated at $37^{\circ} \mathrm{C}$ and $5 \% \mathrm{CO}_{2}$ for 2 hours in order to solidify the solution. For 3D pancreatic differentiation, the authors carried out a five-step protocol based on the method reported by D'Amour et al. in 2006 (7). The islet-like structure showed a robust similarity to adult mouse pancreatic cells in terms of morphology as well as gene expression profile (52). Moreover, more than $50 \%$ of the derived cell clusters showed high insulin expression and secretion of insulin and c-peptide after glucose exposure. Notably, the 3D-differentiated cells exhibited higher insulin secretion (approximately 5-fold higher) than the cells differentiated in the monolayer or 2D platform upon exposure to glucose.

In 2017, Wang et al. generated islet organoids from hESCs under a 3D microenvironment that exhibited biomimetic 3D scaffolds (53). The scaffolds were generated by mixing growth factor-depleted Matrigel with collagen type I solution and mixed with the cell suspension followed by incubation at $37^{\circ} \mathrm{C}$ for 1 hour for solidification. Subsequently, 3D pancreatic 
differentiation was used according to the approach reported by Zhang et al. The generated organoids (3D-derived cell clusters) were composed of pancreatic $\alpha, \beta, \delta$, and PP cells and showed an elevated expression of the transcription factors associated with mature $\beta$ cells, such as Pdx1, Insulin, Glut2, Ngn3, and MafA (53). Moreover, de novo insulin secretion was confirmed by the high expression of the C-peptide.

In 2018, Candiello et al. derived 3D heterogeneous organoids from hESCs using Amikagel hydrogel system (54). Amikagel facilitated the spontaneous aggregation of pancreatic progenitors derived from hESCs and resulted in the formation of homogenous spheroids. In this protocol, hESCs were pre-differentiated into DE and pancreatic progenitor cells based on previous protocols $(55,56)$. Then, only the hESC-derived pancreatic progenitor single cells or the mixture of hESC-derived pancreatic progenitor single cells and HUVECs were plated onto Amikagel-coated plate and incubated for 14 hours to form homogenous and heterogeneous organoids, respectively. Subsequently, the addition of differentiation media to spheroids induced further maturation. The structure as well as the cross-linked moieties in Amikagel played an important role in the maturation of differentiating cells. Furthermore, the unique surface property of Amikagel promoted self-aggregation as well as spontaneous integration of hESC-derived pancreatic progenitor single cells and HUVECs. The hESC-derived pancreatic progenitor spheroids showed upregulation of PDX1 and NKX6.1 and upon maturation, exhibited a high expression of insulin and
C-peptide as well as glucose-responsive insulin secretion (54). In 2019, Tao et al. engineered human islet organoids from hiPSCs-perfused 3D culture using an organ-on-chip platform (57). This platform was based on the design of the multi-layered microfluidic chip device that facilitated circulation of altered media and exposure of organoids to homogenous fluid stress (Fig. 2). In this study, hiPSCs were initially differentiated into embryonic bodies (EBs) followed by endoderm differentiation, islet differentiation and maturation (Fig. 2A). Moreover, real-time imaging as well as investigation of the islet growth was employed with this platform, to demonstrate and the controlled clustering of the EBs formed. The derived organoids exhibited the phenotype and the complexity of human islets and the expression of mature pancreatic $\beta$ cells. Interestingly, the generated organoids showed a marked sensitivity to glucose treatment and high $\mathrm{Ca}^{2+}$ flux release. This study provides an efficient system for the generation of fully mature and functional human islet organoids for further clinical and therapeutic application in human pancreatic disorders and diabetes.

\section{POSSIBLE THERAPEUTIC APPLICATION OF GUT ORGANOIDS IN DIABETES}

Scientific efforts were targeted at the regeneration of damaged islets and the development of an alternative islet. However, gastrointestinal tract (GIT) represents an alternative and renewable source of pancreatic $\beta$ cells for diabetes therapy.

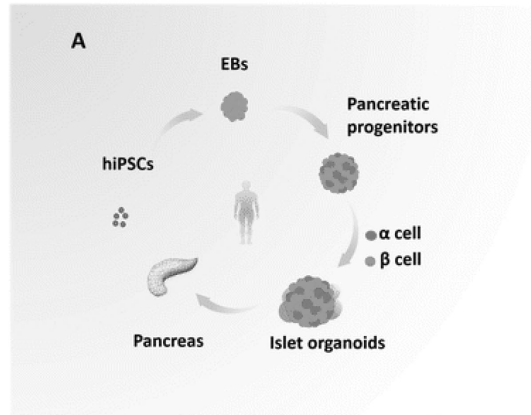

Differentiation of hiPSC-derived islet organoids in vitro

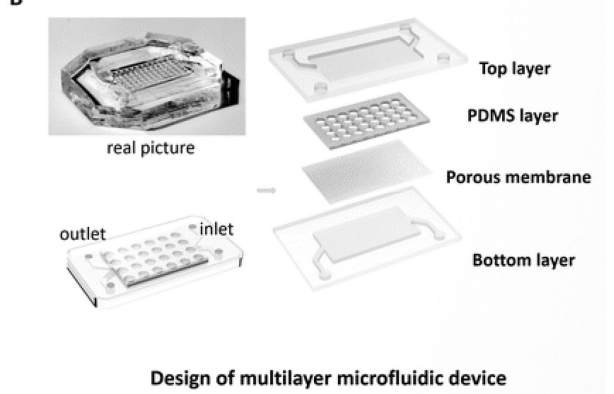

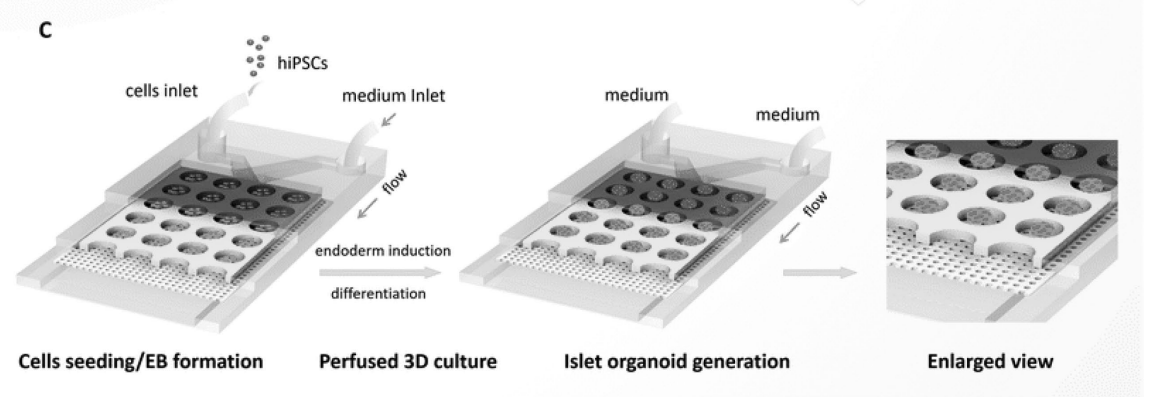

Fig. 2. Representative diagram outlining the procedure for the generation of islet organoids from hiPSCs using a microfluidic device for 3D islet perfusion. (A) In vitro differentiation of hiPSCs into islet organoids. (B) Detailed structure of the multilayer microfluidic chip. (C) Schematic diagram illustrating the steps representing the differentiation of islet organoids on chip and under 3D culture system. This figure is reproduced from Tao et al. 2019 (57) with permission of The Royal Society of Chemistry. 
From a developmental perspective, pancreas and GIT originate in the same gut tube. In particular, stomach- and intestine-derived stem cells yielded a continuous supply of hormone-producing endocrine cells $(58,59)$. The endocrine cells are located only in the islet of Langerhans in the pancreas, but distributed in all the gastric epithelium (60).

In 2014, Chen et al. successfully reprogrammed the intestinal crypt cells into endocrine cells or neo-islets via transduction of key transcription factors (Ngn3, Pdx1, and MafA) into $\beta$ cells (61). The neo-islets manifested the ultrastructure of $\beta$ cells and secreted insulin; further, they showed in vivo modulation of hyperglycemia in diabetic mice. Interestingly, human intestinal organoids transduced with $\beta$ cells transcription factors displayed the features of $\beta$-like cells (61). These findings open vistas for further research into generation of intestinal organoids for insulin production and diabetes therapy on a large scale.

In 2016, the study by Ariyachet et al. concluded that the cells in the antral stomach can be efficiently reprogrammed in vivo with $\beta$ cell factors into insulin-positive cells with a strong functional resemblance to $\beta$ cells (62). Based on comprehensive transcriptional analysis, antral stomach cells shared profound similarities to $\beta$ cells compared with the intestinal enteroendocrine cells. Accordingly, antral stomach was found to exhibit superior potential for $\beta$ cell reprogramming than the other intestinal enteroendocrine cells. Insulin production in stomach-derived cells was maintained for 3 weeks, whereas intestinal enteroendocrine cells showed insulin production for only one week. Interestingly, after abrogation of insulin cells with STZ, GIT-derived insulin cells were regenerated in 3 weeks, which alleviated hyperglycemia $(60,62)$.

The deletion of the transcription factor Foxo1 is another approach for the generation of insulin-secreting cells in gut organoids $(63,64)$. However, other studies demonstrated the key protective role of Foxo1 in the sustained survival of $\beta$ cells $(60,65)$.

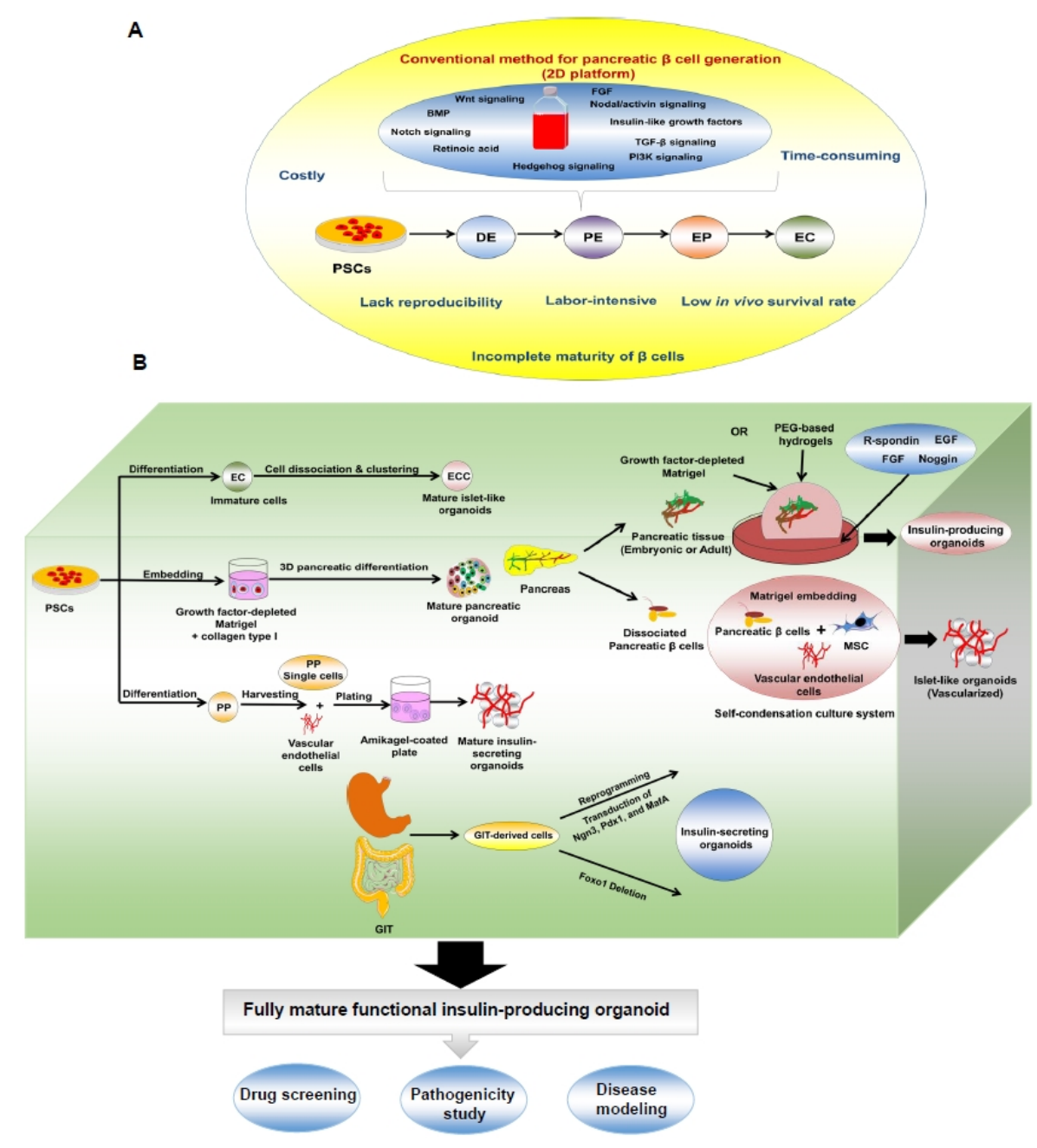

Fig. 3. Schematic diagram illustrating $2 \mathrm{D}$ versus $3 \mathrm{D}$ or organoid platforms for the generation of insulin-secreting islets. (A) Conventional platform for production of insulin-secreting islets with the limitations. (B) Organoid or 3D culture procedures for generating insulin-secreting islet-like organoids from pancreatic tissue, PSCs, and GIT. 
Taken together, these facts and scientific findings pave the way for further studies for practical application of gut organoids generated from patient tissues or PSCs based on their insulin secretion efficacy, and further therapeutic application in diabetes as an alternative source of pancreatic $\beta$ cells.

\section{CONCLUSION}

Recently, various studies reported the generation of functional pancreatic $\beta$ cells from PSCs (ESCS or iPSCs). However, these methods are laborious, time-consuming, expensive, and lack reproducibility (Fig. 3A). Furthermore, many protocols generated immature $\beta$ cells and showed short survival upon in vivo transplantation. Fortunately, the organoid culture or 3D platform represents a promising tool for overcoming the limitations of PSC-derived pancreatic $\beta$ cells. The organoid cultures showed remarkable maturation of the generated pancreatic $\beta$ cells. Pancreatic $\beta$ cell organoids can be generated from isolated pancreatic tissues or from endoderm differentiation of $\beta$ cells derived from PSCs through step-wise protocols (Fig. 3B). The organoid cultures were developed by clustering the cells or embedding them in various matrices, such as Matrigel and synthetic hydrogels. However, the molecular mechanisms of the synthetic matrices, as well as their in vivo validation need to be elucidated in future studies. We also provided a brief overview of the possible reprogramming of GIT-derived cells, in particular, cells in the antral stomach region into insulin-producing $\beta$-like cells (Fig. 3B). Modulation of the expression of factors such as Ngn3, $\mathrm{Pdx} 1$, and MafA may play a key role in reprogramming into $\beta$ cells. Thus far, studies investigating the generation of insulin-producing organoids derived from GIT are preliminary. However, the current findings may encourage scientists in resolving the challenges associated with the generation of insulin-secreting cells from gut organoids, before they can be used as alternative tools for the generation of pancreatic $\beta$ cells for large-scale therapeutic applications.

\section{ACKNOWLEDGEMENTS}

This work was supported by the National Research Foundation of Korea (NRF) grant provided by the Korean government (MSIT) (No. 2017M3A9C6032056) and by the Konkuk University Researcher Fund in 2018.

\section{CONFLICTS OF INTEREST}

The authors have no conflicting interests.

\section{REFERENCES}

1. Posselt AM, Szot GL, Frassetto LA et al (2010) Islet transplantation in type 1 diabetic patients using calcine- urin inhibitor-free immunosuppressive protocols based on T-cell adhesion or costimulation blockade. Transplantation 90, 1595-1601

2. Barton FB, Rickels MR, Alejandro R et al (2012) Improvement in outcomes of clinical islet transplantation: 1999-2010. Diabetes Care 35, 1436-1445

3. Shapiro AMJ, Ricordi C, Hering BJ et al (2006) International trial of the edmonton protocol for islet transplantation. N Engl J Med 355, 1318-1330

4. Russ HA, Bar Y, Ravassard P and Efrat S (2008) In vitro proliferation of cells derived from adult human $\beta$-cells revealed by cell-lineage tracing. Diabetes 57, 1575-1583

5. Gershengorn MC, Hardikar AA, Wei C, Geras-Raaka E, Marcus-Samuels B and Raaka BM (2004) Epithelial-tomesenchymal transition generates proliferative human islet precursor cells. Science 306, 2261-2264

6. Domínguez-Bendala J, Inverardi L and Ricordi C (2011) Stem cell-derived islet cells for transplantation. Curr Opin Organ Transplant 16, 76

7. D'Amour KA, Bang AG, Eliazer S et al (2006) Production of pancreatic hormone-expressing endocrine cells from human embryonic stem cells. Nat Biotechnol 24, 1392

8. Shim J, Kim S, Woo D et al (2007) Directed differentiation of human embryonic stem cells towards a pancreatic cell fate. Diabetologia 50, 1228-1238

9. Shahjalal HM, Dayem AA, Lim KM, Jeon T-i and Cho S-C (2018) Generation of pancreatic $\beta$ cells for treatment of diabetes: advances and challenges. Stem Cell Res Ther 9, 355

10. Rezania A, Bruin JE, Arora P et al (2014) Reversal of diabetes with insulin-producing cells derived in vitro from human pluripotent stem cells. Nat Biotechnol 32, 1121-1133

11. Rezania A, Bruin JE, Riedel MJ et al (2012) Maturation of human embryonic stem cell-derived pancreatic progenitors into functional islets capable of treating pre-existing diabetes in mice. Diabetes 61, 2016-2029

12. Mummery C (2011) Induced pluripotent stem cells-a cautionary note. N Engl J Med 364, 2160-2162

13. Seeberger KL, Dufour JM, Shapiro AMJ, Lakey JR, Rajotte RV and Korbutt GS (2006) Expansion of mesenchymal stem cells from human pancreatic ductal epithelium. Laboratory Investigation 86, 141

14. Todorov I, Omori K, Pascual M et al (2006) Generation of human islets through expansion and differentiation of non-islet pancreatic cells discarded (pancreatic discard) after islet isolation. Pancreas 32, 130-138

15. Cortijo C, Gouzi M, Tissir F and Grapin-Botton A (2012) Planar cell polarity controls pancreatic beta cell differentiation and glucose homeostasis. Cell Rep 2, 1593-1606

16. Dutta D, Heo I and Clevers H (2017) Disease Modeling in Stem Cell-Derived 3D Organoid Systems. Trends Mol Med 23, 393-410

17. Liu C, Oikonomopoulos A, Sayed N and Wu JC (2018) Modeling human diseases with induced pluripotent stem cells: from 2D to 3D and beyond. Development 145, dev156166

18. McCauley HA and Wells JM (2017) Pluripotent stem cell-derived organoids: using principles of developmental 
biology to grow human tissues in a dish. Development $144,958-962$

19. Sato T, Vries RG, Snippert HJ et al (2009) Single Lgr5 stem cells build crypt-villus structures in vitro without a mesenchymal niche. Nature 459, 262

20. Eiraku M, Takata N, Ishibashi H et al (2011) Selforganizing optic-cup morphogenesis in three-dimensional culture. Nature 472, 51

21. Huch M, Dorrell C, Boj SF et al (2013) In vitro expansion of single Lgr5 + liver stem cells induced by Wnt-driven regeneration. Nature 494, 247

22. Lancaster MA, Renner M, Martin C-A et al (2013) Cerebral organoids model human brain development and microcephaly. Nature 501, 373

23. Spence JR, Mayhew CN, Rankin SA et al (2011) Directed differentiation of human pluripotent stem cells into intestinal tissue in vitro. Nature 470, 105

24. Chua CW, Shibata M, Lei M et al (2014) Single luminal epithelial progenitors can generate prostate organoids in culture. Nat Cell Biol 16, 951

25. Dye BR, Hill DR, Ferguson MA et al (2015) In vitro generation of human pluripotent stem cell derived lung organoids. Elife 4, e05098

26. Takasato M, Pei XE, Chiu HS et al (2015) Kidney organoids from human iPS cells contain multiple lineages and model human nephrogenesis. Nature 526, 564

27. Benitez CM, Goodyer WR and Kim SK (2012) Deconstructing pancreas developmental biology. Cold Spring Harb Perspect Biol 4, 1-17

28. Puri S and Hebrok M (2010) Cellular plasticity within the pancreas-lessons learned from development. Dev Cell 18, 342-356

29. Spagnoli FM (2007) From endoderm to pancreas: a multistep journey. Cell Mol Life Sci 64, 2378-2390

30. Pan FC and Wright C (2011) Pancreas organogenesis: from bud to plexus to gland. Dev Dyn 240, 530-565

31. Villasenor A, Chong DC, Henkemeyer $M$ and Cleaver O (2010) Epithelial dynamics of pancreatic branching morphogenesis. Development 137, 4295-4305

32. Seymour PA, Freude KK, Tran MN et al (2007) SOX9 is required for maintenance of the pancreatic progenitor cell pool. Proc Natl Acad Sci U S A 104, 1865-1870

33. Gu G, Dubauskaite J and Melton DA (2002) Direct evidence for the pancreatic lineage: NGN3 + cells are islet progenitors and are distinct from duct progenitors. Development 129, 2447-2457

34. Kopp JL, Dubois CL, Schaffer AE et al (2011) Sox9+ ductal cells are multipotent progenitors throughout development but do not produce new endocrine cells in the normal or injured adult pancreas. Development 138, 653-665

35. Kobberup S, Schmerr M, Dang ML et al (2010) Conditional control of the differentiation competence of pancreatic endocrine and ductal cells by Fgf10. Mech Dev 127, 220-234

36. Hald J, Hjorth JP, German MS, Madsen OD, Serup P and Jensen J (2003) Activated Notch1 prevents differentiation of pancreatic acinar cells and attenuate endocrine development. Dev Biol 260, 426-437

37. Murtaugh LC, Stanger BZ, Kwan KM and Melton DA
(2003) Notch signaling controls multiple steps of pancreatic differentiation. Proc Natl Acad Sci U S A 100, 14920-14925

38. Greggio $C$, De Franceschi $F$, Figueiredo-Larsen $M$ et al (2013) Artificial three-dimensional niches deconstruct pancreas development in vitro. Development 140, 4452-4462

39. Takahashi Y, Takebe T and Taniguchi H (2018) Methods for Generating Vascularized Islet-Like Organoids Via Self-Condensation. Curr Protoc Stem Cell Biol 45, 4

40. Takahashi Y, Takebe T and Taniguchi H (2016) Engineering pancreatic tissues from stem cells towards therapy. Regen Ther 3, 15-23

41. Takebe T, Enomura M, Yoshizawa E et al (2015) Vascularized and Complex Organ Buds from Diverse Tissues via Mesenchymal Cell-Driven Condensation. Cell Stem Cell 16, 556-565

42. Li W, Nakanishi M, Zumsteg A et al (2014) In vivo reprogramming of pancreatic acinar cells to three islet endocrine subtypes. Elife 1, 01846

43. Azzarelli R, Rulands S, Nestorowa S et al (2018) Neurogenin3 phosphorylation controls reprogramming efficiency of pancreatic ductal organoids into endocrine cells. Sci Rep 8, 018-33838

44. Loomans CJM, Williams Giuliani N, Balak J et al (2018) Expansion of Adult Human Pancreatic Tissue Yields Organoids Harboring Progenitor Cells with Endocrine Differentiation Potential. Stem Cell Reports 10, 712-724

45. Zhou Q, Brown J, Kanarek A, Rajagopal J and Melton DA (2008) In vivo reprogramming of adult pancreatic exocrine cells to beta-cells. Nature $455,627-632$

46. Jiang K, Chaimov D, Patel SN et al (2019) 3-D physiomimetic extracellular matrix hydrogels provide a supportive microenvironment for rodent and human islet culture. Biomaterials 198, 37-48

47. Hindley CJ, Cordero-Espinoza L and Huch M (2016) Organoids from adult liver and pancreas: Stem cell biology and biomedical utility. Dev Biol 420, 251-261

48. Morizane R, Lam AQ, Freedman BS, Kishi S, Valerius MT and Bonventre JV (2015) Nephron organoids derived from human pluripotent stem cells model kidney development and injury. Nat Biotechnol 33, 1193-1200

49. Finkbeiner SR, Hill DR, Altheim CH et al (2015) Transcriptome-wide Analysis Reveals Hallmarks of Human Intestine Development and Maturation In Vitro and In Vivo. Stem Cell Reports 3, 00122-00128

50. Shim JH, Kim J, Han J et al (2015) Pancreatic Islet-Like Three-Dimensional Aggregates Derived From Human Embryonic Stem Cells Ameliorate Hyperglycemia in Streptozotocin-Induced Diabetic Mice. Cell Transplant 24, 2155-2168

51. Kim Y, Kim H, Ko UH et al (2016) Islet-like organoids derived from human pluripotent stem cells efficiently function in the glucose responsiveness in vitro and in vivo. Sci Rep 6, 35145

52. Wang $X$ and Ye K (2009) Three-dimensional differentiation of embryonic stem cells into islet-like insulin-producing clusters. Tissue Eng Part A 15, 1941-1952

53. Wang W, Jin S and Ye K (2017) Development of Islet 
Organoids from H9 Human Embryonic Stem Cells in Biomimetic 3D Scaffolds. Stem Cells Dev 26, 394-404

54. Candiello J, Grandhi TSP, Goh SK et al (2018) 3D heterogeneous islet organoid generation from human embryonic stem cells using a novel engineered hydrogel platform. Biomaterials 177, 27-39

55. Jaramillo M, Mathew S, Mamiya H, Goh SK and Banerjee I (2015) Endothelial cells mediate islet-specific maturation of human embryonic stem cell-derived pancreatic progenitor cells. Tissue Eng Part A 21, 14-25

56. Pagliuca FW, Millman JR, Gurtler M et al (2014) Generation of functional human pancreatic beta cells in vitro. Cell 159, 428-439

57. Tao T, Wang Y, Chen W et al (2019) Engineering human islet organoids from iPSCs using an organ-on-chip platform. Lab on a Chip 19, 948-958

58. May CL and Kaestner KH (2010) Gut endocrine cell development. Mol Cell Endocrinol 323, 70-75

59. Schonhoff SE, Giel-Moloney M and Leiter AB (2004) Minireview: Development and differentiation of gut endocrine cells. Endocrinology 145, 2639-2644

60. McCauley HA and Wells JM (2016) Sweet Relief: Reprogramming Gastric Endocrine Cells to Make Insulin. Cell Stem Cell 18, 295-297

61. Chen YJ, Finkbeiner SR, Weinblatt D et al (2014) De novo formation of insulin-producing "neo-beta cell islets" from intestinal crypts. Cell Rep 6, 1046-1058

62. Ariyachet C, Tovaglieri A, Xiang G et al (2016) Reprogrammed stomach tissue as a renewable source of functional beta cells for blood glucose regulation. Cell Stem Cell 18, 410-421

63. Bouchi R, Foo KS, Hua $\mathrm{H}$ et al (2014) FOXO1 inhibition yields functional insulin-producing cells in human gut organoid cultures. Nat Commun 5, 1-11

64. Talchai C, Xuan S, Kitamura T, DePinho RA and Accili D (2012) Generation of functional insulin-producing cells in the gut by Foxo1 ablation. Nat Genet 44, 406-412

65. Talchai SC and Accili D (2015) Legacy Effect of Foxo1 in Pancreatic Endocrine Progenitors on Adult beta-Cell Mass and Function. Diabetes 64, 2868-2879 